\title{
Researching telecare: the importance of context
}

\author{
Kate Hamblin, Sue Yeandle and Gary Fry
}

\begin{abstract}
Purpose - The purpose of this paper is to present a research method which offers insights into the factors which affect the optimal use and implementation of telecare, or which may lead to its rejection by older people with support needs - factors pertinent to those involved in the design and delivery of both telecare research studies and of services.

Design/methodology/approach - The methodology outlined, influenced by Chicago School and Science and Technology Studies, emphasises the importance of context when examining social phenomena, such as the use of technology. The multi-method approach identified key patterns which provide insights into how telecare was used by a sample of older adults, including information on its suboptimal use and rejection.

Findings - The study of telecare use in real-life situations - and the investigation of other complex social interventions - requires an approach which fully considers the importance of context in explaining social phenomena. The main value of the method and findings lies in the insights offered to designers of larger studies which seek to generalise results, including telecare randomised control trials, as well as for those involved in the delivery of telecare services to achieve optimal adoption and use.

Originality/value - The study methods described combined ethnographic, longitudinal and qualitative methodologies and creative research tools in an innovative way to allow exploration of how context affects the uptake and use of telecare.
\end{abstract}

Keywords Interviews, Ethnography, Observations, Science and technology studies, Chicago School, Telecare

Paper type Research paper

\section{Introduction: telecare and the "evidence" debate}

In their paper on methodological approaches to evaluating telecare and telehealth interventions, Davies and Newman (2011) propose a hierarchy of research types, in which they rank "case studies and qualitative research" second lowest, and place randomised control trials (RCTs) just beneath the pinnacle of the systematic review. When the project that is the focus of this paper began, the research team were often told that there was "no evidence" of the effectiveness or usefulness of telecare. At that time, findings from the largest RCT of telecare in the UK (the Whole System Demonstrator project[1]) were not available and most research on telecare came from small-scale, qualitative or pilot studies, some undertaken by telecare providers. Such research was not considered rigorous enough to draw conclusions, and calls for more "robust" evidence persisted (ADASS, 2014).

It has been argued, however, that the lack of "evidence" about the effectiveness of telecare is a matter of disciplinary politics in a multidisciplinary field characterised by "territorial disputes about whose knowledge is authoritative" (Williams et al., 2003, p. 52). Avoiding setting different approaches against one another, this paper argues that, while qualitative methods offer an alternative, insightful approach in their own right, they also provide essential information, both for the design of RCTs and other large-scale research, and for those commissioning and delivering telecare services. Positivistic research can obscure the complexity and context within which the devices involved are situated (Greenhalgh and Swinglehurst, 2011); indeed, qualitative interviews with service users who declined to participate in, or withdrew from, the Whole Systems
Kate Hamblin is a Senior Research Fellow at the Oxford Institute of Population Ageing, University of Oxford, Oxford, UK. Sue Yeandle is a Professor of Sociology at the CIRCLE, University of Sheffield,

Sheffield, UK.

Gary Fry is based at the School of Dementia Studies, University of Bradford, Bradford, UK.

Received 25 April 2017

Revised 3 July 2017

Accepted 9 July 2017

(C) Kate Hamblin, Sue Yeandle and Gary Fry. Published by Emerald Publishing Limited. This article is published under the Creative

Commons Attribution (CC BY 4.0)

licence. Anyone may reproduce,

distribute, translate and create

derivative works of this article

(for both commercial \&

non-commercial purposes),

subject to full attribution to the

original publication and authors.

The full terms of this licence may be seen at http://creativecommons.org/ licences/by/4.0/legalcode

The authors thank the Technology Strategy Board (TSB), the Economic and Social Research Council (ESRC) and the National Institute for Health Research (NIHR) for funding the research (Project No. 2592-25185) on which this paper is based, and the wider project consortium for their contributions. In addition, the authors thank Dr Emanuela

Bianchera, Dr Chrissy Buse and Dr Emma-Reetta Koivunen for their contributions to the research design and fieldwork and Professor Pete Buckle for his comments on earlier drafts of this paper. The authors also offer their thanks to the research participants for their generosity and insights. 
Demonstrator Sites project found that many complex factors influenced their rejection of telecare (Sanders et al., 2012) - factors which designers of RCTs and of telecare services should consider when assessing the impacts of these devices. This paper presents a multi-method approach to the study of telecare; this could also be applied to the investigation or delivery of other complex social interventions where context and social interactions play a role in shaping outcomes. We first outline a methodology utilised by the (Aktive) project, designed to capture the complexities of the context within which telecare devices were situated, then we use research findings to demonstrate the importance of this context in mediating the adoption and use of telecare.

\section{Telecare research: an alternative approach}

It is widely accepted that research methods should be selected on the basis of their suitability for examining a particular phenomenon. In health and social care settings, experimental and quasi-experimental research designs are traditionally seen as the "gold standard", producing robust, generalisable findings. However, it has been argued (Morgan-Trimmer and Wood, 2016) that such studies adopt a linear approach and aim to establish relationships between variables, and do not analyse how people's responses to interventions are mediated by prior experiences, attitudes or by the interplay of different social actors in the situation. This, and the distance between the researcher and the data, underpins speculation about why particular outcomes occurred or an intervention was successful (or not) (Morgan-Trimmer and Wood, 2016). When an experimental or quasi-experimental approach to telecare research is used, the technology tends to be conceptualised as an objective, predictable phenomenon, ignoring the role of the user and the context; both affect outcomes and the technology itself (Williams and Edge, 1996). By contrast, the approach adopted in science and technology studies (STS) conceptualises technology as a social phenomenon which cannot be studied in isolation from its social context. STS emerged in the 1960s, arguing that technology both shapes (making some things possible, or impossible) and is shaped by (humans can engage with, and can disable/enable its functions) context and interactions (Greenhalgh and Swinglehurst, 2011; Williams and Edge, 1996). STS is situated within the "contextualist paradigm" advocated by the Chicago School sociologists, who argued that "one cannot understand social life without understanding the arrangements of particular social actors in particular social times and places [...] Nothing that ever occurs in the social world occurs "'net of other variables'” (Abbott, 1997, p. 1152). The STS approach to data collection is flexible, responsive, iterative and does not provide a "snapshot" of one point in time. It can also help explain why expected and actual outcomes are not aligned (Greenhalgh and Swinglehurst, 2011). This contextualist approach cannot identify the importance of specific "crucial variables", but can reveal patterns of action likely to occur in similar contexts. The identification of these patterns, through the examination of the context within which a complex intervention such as telecare is situated, is, we will argue, an important stage in the design of any research, including RCTs, and can assist those designing and delivering services[2] to ensure they are provided in a way that promotes optimal usage and outcomes.

The on-going search for an appropriate methodology for the study of how older people (and others) use telecare needs also to recognise that, as an intervention, telecare has many of the features of the "complex social programmes" described in discussions of evidence-based policymaking: it involves a "long implementation chain", with many and varied stakeholders and end users; it is embedded in complex and divergent social systems; it is "implemented amid the turbulence of other interventions"; and it is introduced and overseen by practitioners who "work constantly to improve the delivery of interventions", disrupting uniformity of implementation in the process (Pawson et al., 2011, p. 519). The range of actors likely to interact with telecare is large, and telecare systems are both extremely complex and continuously in flux at the macro level (in England, for example, local authority telecare services are typically recommissioned every few years) and at the micro level (individual telecare users' needs are likely to be reassessed annually, or when an individual's circumstances change) (Yeandle et al., 2014; Buckle, 2014). Furthermore, "telecare" is not a single product, but an array of equipment which has emerged across different "generations" of product development (Scottish Government, 2008). Even if a single device (e.g. a personal "pendant alarm", of the type worn continuously on the body, requiring the user to

PAGE 76 JOURNAL OF ENABLING TECHNOLOGIES $\mid$ VOL. 11 NO. 32017 
press a button to send an alert and linked to a remote monitoring centre) is isolated to evaluate its effects, in reality the device is likely to be delivered to users in many, and variable, ways by a variety of providers, including (often) a mix of third sector, private and local authority organisations in different localities, in a variety of combinations with other services (De Leonibus et al. , 2013). Supply and charging policies vary, too; in some areas local authorities provide a telecare service as a free service, while others levy fees (e.g. both examples were seen in AKTIVE). Telecare users' lives are also complex, "messy" and in flux, and research needs to reflect and capture this complexity, rather than impose artificial "order", and seek to draw conclusions applicable to wider contexts.

The perspectives of STS and the Chicago School suggest that the methods used to explore telecare as a subset of technology need to be capable of exploring the contexts and social interactions in which it is embedded (Greenhalgh and Swinglehurst, 2011). This paper describes a methodology developed to explore these contextual factors, which shape users' experiences and have the potential to "disrupt" the performance of telecare interventions. This complexity, the study revealed, has implications for health and social care professionals working with telecare (Sanders et al., 2012); for researchers planning experimental or quasi-experimental studies of telecare (whose protocols should take account of this), and for commissioners, manufacturers and suppliers, who need an understanding of the real world context in which telecare is provided to assess the advantages and disadvantages of introducing it.

\section{Everyday life analysis (ELA): bringing the social context back in}

The aim of the AKTIVE was to develop new knowledge relating to the use of telecare and to the barriers affecting its uptake and successful use[3]. The research design included a literature search (AKTIVE Consortium 2013) and preliminary interviews (Yeandle et al., 2014), which showed that different stakeholders (policy-makers, telecare commissioners, academics, care workers and unpaid carers) had varied perspectives on the purpose of telecare. Taking account of this, the research team sought to include the views of both older telecare users and those involved in their care (e.g. care workers or carers) in the central element of the study, its ELA visits to the homes of older people who had been supplied with telecare equipment as research within the contextualist paradigm suggests that the perspectives of users, and those involved in their care, may be critical to understanding barriers to telecare adoption and use and the next section outlines our method and approach.

Our fieldwork targeted people aged 65+ who had been assessed as "susceptible to falls", or had a diagnosis (or were displaying some symptoms) of cognitive impairment, or both. Our data collection strategy involved visiting each participant four to six times over a period of six to nine months, typically with about four to six weeks between visits. Recruitment was arranged in partnership with two English local authorities[4]. The research design enabled any family members, friends, neighbours, carers or care workers providing support to be included (with the older person's consent), providing additional contextual information. The final sample comprised 60 participants who remained in the study long enough for longitudinal analysis; 16 were in both the "falls" and "cognitive impairment" categories; 35 were in the former only; and nine were in the latter only.

By taking important contextual information into account, critical observations can provide insight into causal chains (Scriven, 2008); further, ethnographic studies often require researchers to embed themselves in the field for an extended period to observe interactions (Morley and Silverstone, 1991). Based on established methodological guidance (Silverstone et al., 1991), tools were developed for the specificity of the research context, and key ethnographic principles - observing and noting "routine" behaviour in "natural settings" and drawing inferences (Lull, 1987) - were adopted. The approach involved a carefully applied ethical framework designed to avoid intrusiveness or upsetting the older people studied, many of whom were aged 85 or older, and living alone. Ethnography has become an established methodology within telecare research (cf. Greenhalgh and Swinglehurst, 2011; Mair et al., 2008: Pols and Willems, 2011; Milligan et al., 2011). The study team's ELA visits varied in length (averaging about an hour) and were used to observe participants in various settings in and around their homes and local areas, as they negotiated their surroundings and engaged in everyday activities (such as making tea or 
walking their dog). Four field researchers made, in total, over 400 ELA visits; in each case the older person studied was visited by the same researcher. An observational template was used to capture: researchers' thoughts prior to each research visit; what happened during the visit; and to ensure issues discussed or observed were reported, including how the telecare was being used and any issues not apparent from recorded conversation during the visit, such as relevant features of their home; and dynamics observed between persons present during the visit.

The interview approach chosen for the "guided conversations" (Downs, 1997) with participants generated data on participants' attitudes, opinions and activities. As part of the longitudinal research design, different "topic guides" were developed for each visit to help explore participants' everyday lives and to encourage a focus on the life course. The first ELA session with each participant focused on installation of the telecare equipment, the rationale for acquiring it and explored participants' biographies, aiming to give them the freedom to "dictate those events and experiences that have determined the course of their lives" (Bornat and Bytheway, 2010, p. 6). For subsequent visits, the topic guides (covering social networks; homes and environments; education and employment histories; health and well-being; and attitudes towards technology) were tailored to individual participants' experience, and updates on events since the previous visit were collected, with particular reference to use of, and experiences with, the telecare equipment. While six to nine months is a relatively "compressed" timeframe for longitudinal research, it worked well in a study of older people with impairments and mobility limitations, and was adequate and appropriate for the study objectives, research questions and conceptual framework. Most research visits were conducted when the older person was alone, but some took place when a family/friend carer, relative or care worker was present, sometimes creating joint interviews.

Within this multi-method approach, and where appropriate, participants were offered an "ELA box", tailored to their circumstances, typically including a notebook, a disposable camera, and paper and stamped-addressed envelopes (for writing to researchers). By individually tailoring the ELA boxes, the researchers could also tactfully navigate issues relating to literacy or to physical/sensory disabilities. These creative methods were inspired by their effective use in team members' previous research, and similar approaches have been used in other telecare research (Wherton et al., 2012). ELA participants were given flexible instructions to support completion of their diaries and on taking photographs focused on their home, relationships, activities and any incidents involving their telecare devices. There was variation in the information recorded in the diaries, with some recording their daily activities, concerns and feelings at length, while others made bullet point-style notes; both formats provided insight into their daily lives and use of telecare. The photographs were printed and discussed with the participants at the next ELA visit.

\section{Telecare acceptance and use: the importance of context}

The ELA method provided crucial information relevant to the efficiency and effectiveness of each specific telecare intervention or package, and patterns observed in the data were used to identify what "successful" use of telecare was, and what it meant, both to those using the equipment and those supporting them. Their criteria for success often differed from those used by professionals/commissioners in the health and social care system, but paying attention to older people's perceptions, and to the contextual issues which affected their interactions with telecare (and other outcomes) generated important insights. Using STS-influenced ethnographic methods thus helped us to explain why expected and actual outcomes for telecare often do not align (Greenhalgh and Swinglehurst, 2011). The following section showcases the data collected using the ELA methodology, demonstrating that patterns of behaviour and attitudes related to telecare interventions are complex, and spatio-temporally located, enabling us to draw inferences capable of being tested in other contexts.

During the study, the research team rarely observed, or heard about, failures of telecare devices caused by mechanical problems with the product. When used as intended, devices did not trigger an alert to the designated response centre/responder. Study participants often mentioned incidents in which they could have used their telecare devices (especially pendant alarms), but had instead opted not to activate them to summon help. This "rejection" of the devices (either total or partial), and why it occurred, is relevant to the appraisal of telecare as "successful" 
or "unsuccessful", as without such understanding it is hard to assess the impact or effectiveness of telecare- here the ELA method proved invaluable.

Those who retained their devices, but did not use them as expected or directed, tended to distinguish between different types of fall: falls they could recover from alone (often with substantial effort or long periods on the floor), and those for which they needed their pendant alarm to get help. The decision to activate the alarm was mediated by several important contextual factors. Those who had not previously activated their device spoke of feeling confused about would happen: would they need to get to the response box to give instructions? Who would be summoned to help them - would it be a carer/neighbour/family member, the police, an ambulance or an emergency response service? These uncertainties, plus a desire to choose a response they felt suitable to the situation (e.g. not a family member late at night; not a "stranger" if security was a concern), could give participants pause, or deter their use of the pendant. Although these issues had usually been discussed when the equipment was installed, users and those caring for them explained that this had often been at a time of heightened stress, linked to discharge from hospital or living alone for the first time after the death of a spouse, and for many had been the first time they had been in contact with social services. In these circumstances, instructions were easily misunderstood or forgotten, and some had felt overwhelmed by visits from different services, so found it hard to disentangle who they should contact for advice. Some of the participants were inducted into the study when their telecare devices were installed. The research team was therefore able to observe the advice and information they received. There were a few instances in which the information provided was unclear or incorrect (e.g. whether the device could be worn in the shower, the cost of the service and eligibility criteria for funded telecare). The assessors, installers, monitoring and response centre staff were therefore all an important part of the context the telecare was situated in, and as such affected how participants viewed and used their devices. This embeddedness in social contexts meant that devices were not neutral, predictable phenomena whose outcomes could easily be captured in isolation.

Among those who had previously activated their pendant or other telecare device, past experience affected their later decisions. Some had found their previous experience negative and abandoned their device(s) to a drawer or other "safe place". In some cases the previous response had surprised them - they had expected a family member to attend, but a response service came instead; for others, a long wait time had left them distrustful and reluctant to activate their alarm. By collecting data about these experiences longitudinally, the researchers could capture changing attitudes towards the telecare devices over time, including, in a few cases, their removal following false alerts or if the cost of the service was felt to be too significant.

Broader contextual issues also affected successful use of telecare. The study had the flexibility to capture perspectives of people within an older person's caring network (Yeandle, 2014) and these showed the importance of the interpersonal relationships within which telecare was situated; the participants were keen not to be "burdensome" to those who provided support by activating their device too regularly or at particular times. Telecare was often initially installed (and accepted by participants) because it offered reassurance to those supporting them, but this had to be balanced with their concerns about actually using their devices. Tensions could arise about wearing and using the device between the older person and those providing their care (including, in some cases, care workers attending them at home); others reported that their telecare device(s) had improved relationships by providing reassurance between visits and forging stronger links between neighbours listed as responders.

The ELA method, and its emphasis on participants' life histories, gave research participants an opportunity to reflect on their biographies, exploring how their past and present identities were bound up with roles they had taken in life. When telecare was installed as a response to a sudden event (such as a fall or prior to hospital discharge) some participants experienced considerable change. Some felt it was "out with the old" (activities and interests), and "in with new" (interventions and aids, introduced to reduce risk and provide assistance). Not all were happy about or accepting of these developments and this was especially acute for those who felt they had had little say in the decision making involved. However, well-intentioned changes to their homes had been, or indeed how useful they were, some felt adaptions and the introduction of 
telecare devices or other aids were markers of "old age". Some thought telecare devices worn on the body were unsightly, or not in keeping with how they wished to present themselves, and preferred to keep devices such as pendant alarms "within reach" (e.g. on walking frames or tables, or worn concealed under clothing) which, in reality, made them potentially difficult to activate in an emergency.

Others viewed telecare much more positively, arguing that it provided the vital "back-up" they needed to feel confident about living independently in the community (or being "allowed" by others to continue doing this). As their bodily frailty had increased, many participants had identified areas of their homes as potentially "risky" or "out of bounds"; for some, telecare enabled these "high risk" areas, such as gardens, staircases and upper floors, to remain accessible. It also meant they felt safer and more secure in their homes (especially if living alone) and gave reassurance to their families and friends, often impacting positively on family relationships. Control, and the degree to which the older person felt empowered in decisions about care and support, were critical in shaping their perspectives about telecare.

The ELA method in AKTIVE thus highlighted how context affects the lived experience of telecare and other technologies and changes designed to support older people who become frail. It demonstrated the significance of choice and control when packages of support, including telecare, are developed, and revealed the "trade-off" between how telecare can make people feel and its capacity to help them continue activities which help them to be and feel active, independent and embedded in valued places and communities. When introduced as part of wider (often unwelcome) changes in their lives or homes (home care, home adaptations, the identification of some areas as "risky"), telecare could be hard to reconcile with valued identities as independent and active persons, however.

\section{Strengths and limitations of the ELA approach}

As methods which embrace ethnography and social constructivist approaches generate data very different from those collected in experimental/quasi-experimental research, to compare their "quality" would be to compare apples and oranges. That said, the ELA approach produced valid data, collected in naturalistic settings which could be confirmed in the repeat nature of the visits and, in relevant cases, from others involved in the older person's life and/or care. Our findings were context specific, but enabled patterns and common behaviours or attitudes to be observed, and used to generate theories and hypotheses.

The longitudinal aspect of the ELA method allowed the researchers to observe change over time and ensured they did not merely capture "one-off" events; it was also flexible enough to ensure the researchers were not closed off to "unexpected" data (Silverstone et al., 1991). Spreading research contact over an extended period also meant data could be collected in a less intrusive and intense manner, appropriate for the study's frail older participants, and allowed researchers to factor in "good" and "bad" days, which was particularly important for respondents whose health was variable, or who had cognitive impairment. The ELA visits also allowed the researchers to build trust and rapport, facilitate understanding and create opportunities to clarify material discussed in earlier visits. Ethnographic interviews yielded much information about participants' use of and feelings about telecare, but also provided insights into the everyday lives of older people. The participants could also be observed demonstrating how they used their telecare devices, sometimes with great difficulty, and researchers could note how their homes had changed over time, as frailty increased or, in some cases, devices and aids were removed as they recovered.

Reflecting on study visits, researchers felt the combination of open, "conversational" questions and additional creative exercises and techniques enabled most participants (sometimes with the assistance of their carers) to voice their opinions about telecare, as well as to discuss past and present events and share their general concerns and aspirations. The ELA topic guides allowed researchers to explore older people's lives in ways which were enjoyable for both parties and which produced material relevant to the study, although, due to the adaptable nature of the guides, it could sometimes be challenging to keep participants "on topic". Where applicable, the joint interviews also produced insight into interpersonal dynamics, 
interactions and relationships (Arksey, 1996). Some carers were able to re-word questions or comments, helping with comprehension, but joint interviews could also be challenging if carers tried to "take over" the conversation, a particular problem in one case where the participant had dementia. The research team used techniques such as redirecting questions to the older person (even if the other person present had already responded to it). If carers "spoke over", answered on behalf of the older person, or corrected them to provide what they felt were the "facts" (Pratt, 2002), the researcher suggested an additional, separate interview with the carer, to enable them to tell "their side of the story" without devaluing or demoralising the older person.

The creative aspects of the ELA methodology were used by many, though not all participants (in total, 21 participants used diaries and eight took photographs). Diaries, frequently used in social science and health research to record time use, social interaction and perceptions of health over time, have some limitations, as participants can feel uneasy about recording personal thoughts, or may lack confidence in their ability to write in a "correct" way (Välimäki et al., 2007). To mitigate this risk, the guidance was flexible in its approach, suggesting topics for notes which could be expressed in whatever format the participant felt comfortable with. The diaries were valuable as they relied on short-term memory and reduced recall errors, and allowed the researchers to triangulate the data. Research visits often began with the participant - particularly if they were unable to leave their home alone - stating that "nothing had happened" since the previous visit. However the diaries often sparked memories, clarifying points which had become confused, or provided insight into the participants' perceptions. They also kept the participants engaged with the project between visits and could prompt the participants to feel a sense of achievement afterwards, and provided the opportunity for the participants to "say" things they perhaps felt uncomfortable about mentioning to the researcher. Fewer participants used photographs, and the cameras raised some practical issues for the research team due to difficulties in getting photos developed quickly enough for the pictures to still resonate with the participants. Those who did take photographs tended to focus on three aspects of their everyday experience: the people in their lives; places (typically their home or localities they visited); and things which helped them, including their telecare devices and other equipment.

The ELA method produced a large volume of data, comprising approximately 400 transcripts in addition to fieldwork notes, and the diary and photographic data. This potentially overwhelming amount of data were managed using an analytical strategy which employed thematic analysis (identified and cross-checked by all researchers) and computer-aided techniques for data mark-up and retrieval, plus various indexing and summarising techniques to help the whole team acquire and retain a "picture" of each participant, including those they had not personally met. In line with an STS and contextualist approach, the analysis focused on the processes at work, as opposed to the relationships between, or the relative importance of, variables; the focus was therefore on identifying patterns of actions which could be applied to similar contexts (Abbott, 1997). The analysis was nonetheless a challenging task, and required more time than originally allocated to it in the project plan. Due to the qualitative nature of the study, a representative sample of the wider population of telecare users was not the aim[5]. It could be argued, therefore, that this limits the generalisability of the findings, yet though the findings could be context specific, the in-depth methodology provided a valuable "thick description" and valid picture of the use of telecare by older people in these two authorities, and the patterns we observed can be explored elsewhere and used to generate theory.

\section{Conclusion}

This paper has described an innovative methodological approach applied in telecare research. The dominant methodological debate in this field has been inclined towards RCTs as the "gold standard" for "robust evidence". Telecare is a complex social intervention, however, affected by a myriad of confounding variables which may influence outcomes and compromise measurement of effects. A multidisciplinary and longitudinal approach is thus essential to fully address the importance of context in mediating the experience and use of telecare. 
The research team developed a methodology to permit careful observation of the biographical and social context in which the telecare equipment was situated, and to examine its effect on how users and those involved in their care perceived and engaged with it. Influenced by STS and the Chicago School, the methods chosen for the ELA were primarily ethnographic observations and interviews with a biographical or thematic focus; these were supplemented by photographs and diaries to produce a nuanced overview of older people's everyday lives and of the role of telecare within them.

While labour-intensive in terms of data collection and analysis, the ELA approach yielded important insights which other methods could not have achieved, including data that highlighted the importance of the context within which the telecare was situated. They also provided understanding of broader issues related to ageing, including challenges to identity, the impact of physical or mental health problems, changes in relationships, care and support and social isolation. Understanding of these issues is very important for interpreting the factors which affect how telecare devices are received and used, with implications for whether these can be judged "successful" or worth investing in. The ELA method, with its emphasis on each participant's "life history" in the first session, offered research participants an opportunity to reflect on their biographies, exploring how their past and present identities were bound up with roles they had taken in life. Many research participants felt "lost" within their social relationships as they struggled to shift their identity from "carer" to "cared for"; many expressed a desire not to become a "burden" to their families. It was often within these contexts, with painful changes on-going, that telecare was introduced. Few in the sample had telecare installed as a preventative measure (although a small minority acquired it as standard when entering supported housing); it was generally installed in response to a sudden event such as a fall, loss of a co-resident spouse or hospitalisation, and as a result, some participants felt upset or unsettled. These feelings affected their interactions with their telecare devices. For those who felt control over their daily lives and living environment had been taken away, the decision about whether to wear, or to activate, their devices was significant.

These are important issues. They mediate the use of telecare devices by older adults, and need to be considered both in the design of research to evaluate its outcomes, and by those developing and delivering telecare services and products to promote their optimal use. For example, a RCT study could be designed to take adequate account of the context in which telecare is installed, and to ensure that, prior to the assessment of outcomes, it is introduced and delivered in a manner broadly comparable across the intervention group.

\section{Notes}

1. The Whole Systems Demonstrator is the largest RCT of telecare and telehealth conducted in the UK to date, although other studies had been conducted in the years prior to its launch (e.g. Opening Doors for Older People, Bowes and McColgan, 2006).

2. See (Hamblin, 2017).

3. More information on the AKTIVE, including the working papers can be found here: www.aktive.org.uk

4. Recruitment support was also provided by the Thames Valley Dementia and Neurodegenerative Diseases Research Network and the Oxford Health NHS Foundation Trust.

5. An accurate picture of this wider population was not possible, as the data collected by the local authorities on their telecare users did not permit this.

\section{References}

Abbott, A. (1997), "Of time and space: the contemporary relevance of the Chicago School", Social Forces, Vol. 75 No. 4, pp. 1149-82.

ADASS (2014), "Better Care Technology Survey 2014 Report", ADASS, London.

AKTIVE Consortium (2013), "The role of telecare in meeting the care needs of older people: themes, debates and perspectives in the literature on ageing and technology", AKTIVE Research Report Vol. 1, CIRCLE, University of Leeds, Leeds.

PAGE 82 JOURNAL OF ENABLING TECHNOLOGIES $\mid$ VOL. 11 NO. 32017 
Arksey, H. (1996), "Collecting data through joint interviews", Social Research Update, Vol. 15, pp. 1-4.

Bowes, A. and McColgan, G. (2006), Smart Technology and Community Care for Older People: Innovation in West Lothian, Age Concern Scotland, Edinburgh.

Bornat, J. and Bytheway, B. (2010), "Perceptions and presentations of living with everyday risk in later life", British Journal of Social Work, Vol. 40 No. 4, pp. 1118-34.

Buckle, P. (2014), "Human factors that influence the performance of the telecare system", AKTIVE research report, Working Paper No. 7, Vol. 2, University of Leeds, Leeds.

Davies, A. and Newman, S. (2011), "Evaluating telecare and telehealth Interventions", WSDAN briefing paper, Kings Fund, London.

De Leonibus, V., Bartosova, R. and Lewis, E. (2013), "ALT market in the UK - AKTIVE market report: initial overview", AKTIVE research report, CIRCLE, University of Leeds, Leeds.

Downs, M. (1997), "The emergence of the person in dementia research", Ageing and Society, Vol. 17 No. 5 , pp. 597-607.

Greenhalgh, T. and Swinglehurst, D. (2011), "Studying technology use as social practice: the untapped potential of ethnography”, BMC Medicine, Vol. 9 No. 45, pp. 1-7.

Hamblin, K. (2017), "Telecare, obtrusiveness, acceptance and use: an empirical exploration”, British Journal of Occupational Therapy, Vol. 80 No. 2, pp. 132-8.

Lull, J. (1987), World Families Watch Television, Sage, Newbury Park, CA.

Mair, F.S., Hiscock, J. and Beaton, S.C. (2008), "Understanding factors that inhibit or promote the utilization of telecare in chronic lung disease", Chronic Illness, Vol. 4 No. 2, pp. 110-17.

Milligan, C., Roberts, C. and Mort, M. (2011), “Telecare and older people: who cares where?”, Social Science \& Medicine, Vol. 72 No. 3, pp. 347-54.

Morgan-Trimmer, S. and Wood, F. (2016), "Ethnographic methods for process evaluations of complex health behaviour interventions”, Trials, Vol. 17 No. 232, pp. 1-11.

Morley, D. and Silverstone, R. (1991), "Communication and context: ethnographic perspectives on the media audience", in Jankowski, N.W. and Jensen, K. (Eds), A Handbook of Qualitative Methodologies for Mass Communication Research, Routledge, London, pp. 149-62.

Pawson, R., Wong, G. and Owen, L. (2011), "Known knowns, known unknowns, unknown unknowns: the predicament of evidence-based policy”, American Journal of Evaluation, Vol. 32 No. 4, pp. 518-46.

Pols, J. and Willems, D. (2011), "Innovation and evaluation: taming and unleashing telecare technology", Sociology of Health \& IIIness, Vol. 33 No. 3, pp. 484-98.

Pratt, R. (2002), "Nobody's ever asked how I felt”, in Wilkinson, H. (Ed.), The Perspectives of People with Dementia: Research Methods and Motivations, Jessica Kingsley Publishers, London, pp. 165-82.

Sanders, C., Rogers, A., Bowen, R., Bower, P., Hirani, S., Cartwright, M., Fitzpatrick, R., Knapp, M., Barlow, M., Hendy, J., Chrysanthaki, T., Bardsley, M. and Newman, S. (2012), "Exploring barriers to participation and adoption of telehealth and telecare within the whole system demonstrator trial: a qualitative study", BMC Health Services Research, Vol. 12 No. 1, p. 220.

Scottish Government (2008), Seizing the Opportunity: Telecare Strategy 2009-2010, Scottish Government, Edinburgh.

Scriven, M. (2008), "A summative evaluation of RCT methodology: \& an alternative approach to causal research", Journal of Multidisciplinary Evaluation, Vol. 5 No. 9, pp. 11-24.

Silverstone, R., Hirsch, E. and Morley, D. (1991), "Listening to a long conversation: an ethnographic approach to the study of information and communication technologies in the home", Cultural Studies, Vol. 5 No. 2, pp. 204-27.

Välimäki, T., Vehviläinen-Julkunen, K. and Pietilä, A.M. (2007), "Diaries as research data in a study on family caregivers of people with Alzheimer's disease: methodological issues", Journal of Advanced Nursing, Vol. 59 No. 1, pp. 68-76.

Wherton, J., Sugarhood, P., Procter, R., Rouncefield, M., Dewsbury, G., Hinder, S. and Greenhalgh, T. (2012), "Designing assisted living technologies 'in the wild': preliminary experiences with cultural probe methodology", BMC Medical Research Methodology, Vol. 12 No. 188, pp. 1-13. 
Williams, R. and Edge, D. (1996), "The social shaping of technology", Research Policy, Vol. 25 No. 6, pp. 865-99.

Williams, T., May, C., Mair, F., Mort, M. and Gask, L. (2003), "Normative models of health technology assessment and the social production of evidence about telehealth care", Health Policy, Vol. 64 No. 1, pp. 39-54.

Yeandle, S. (2014), "Frail older people and their networks of support: how does telecare fit in?", AKTIVE research report, Vol. 2, Working Paper No. 2, University of Leeds, Leeds.

Yeandle, S., Buckle, P., Fry, G., Hamblin, K., Koivunen, E.-R. and McGinley, C. (2014), "The AKTIVE project's social, design and prospective hazard research: research methods", AKTIVE research report, Vol. 3, Leeds: CIRCLE, University of Leeds, Leeds.

\section{Corresponding author}

Kate Hamblin can be contacted at: kate.hamblin@ageing.ox.ac.uk

For instructions on how to order reprints of this article, please visit our website: 